\title{
Analysis of the cytotoxic effects, cellular uptake and cellular distribution of paclitaxel-loaded nanoparticles in glioblastoma cells in vitro
}

\author{
LIN WANG $^{1}$, CHUNHUI LIU ${ }^{1}$, FENG QIAO ${ }^{1}$, MINGJUN LI ${ }^{1}$, \\ HUA XIN $^{1}$, NAIFENG CHEN ${ }^{2}$, YAN WU ${ }^{3}$ and JUNXING LIU ${ }^{2}$ \\ ${ }^{1}$ Clinical Laboratory, The First Affiliated Hospital of Jiamusi University, Jiamusi, Heilongjiang 154003;
${ }^{2}$ Department of Pathology and Physiology, School of Basic Medical Sciences of Jiamusi University,
Jiamusi, Heilongjiang $154007 ;{ }^{3}$ Division of Nanomedicine and Nanobiology,
National Center for Nanoscience and Technology, Beijing 100190, P.R. China
}

Received March 20, 2019; Accepted September 18, 2020

DOI: $10.3892 /$ etm.2021.9723

\begin{abstract}
Glioblastoma is the most common and aggressive type of brain tumor. Although treatments for glioblastoma have been improved recently, patients still suffer from local recurrence in addition to poor prognosis. Previous studies have indicated that the efficacy of chemotherapeutic or bioactive agents is severely compromised by the blood-brain barrier and the inherent drug resistance of glioblastoma. The present study developed a delivery system to improve the efficiency of delivering therapeutic agents into glioblastoma cells. The anticancer drug paclitaxel (PTX) was packed into nanoparticles that were composed of amphiphilic poly ( $\gamma$-glutamic-acid-maleimide-co-L-lactide)-1,2-dipalmit oylsn-glycero-3-phosphoethanolaminecopolymer conjugated with targeting moiety transferrin (Tf). The Tf nanoparticles (Tf-NPs) may enter glioblastoma cells via transferrin receptor-mediated endocytosis. MTT assay and flow cytometry were used to explore the cytotoxic effects, cellular uptake and cellular distribution of paclitaxel-loaded nanoparticles. The results indicated that both PTX and PTX-Tf-NPs inhibited the viability of rat glioblastoma C6 cells in a dose-dependent manner, but the PTX-Tf-NPs exhibited a greater inhibitory effect compared with PTX, even at higher concentrations
\end{abstract}

Correspondence to: Professor Junxing Liu, Department of Pathology and Physiology, School of Basic Medical Sciences of Jiamusi University, Basic Medicine Building, 148 Xuefu Street, Jiamusi, Heilongjiang 154007, P.R. China

E-mail: liujunxing0982@163.com

Professor Yan Wu, Division of Nanomedicine and Nanobiology, National Center for Nanoscience and Technology, 11 Beiyitiao Zhongguancun, Beijing 100190, P.R. China

E-mail:wuy@nanoctr.cn

Key words: glioblastoma, nanocarrier, nanoparticles, transferrin, targeting moiety
(0.4, 2 and $10 \mu \mathrm{g} / \mathrm{ml})$. However, both PTX and PTX-Tf-NPs exhibited a reduced inhibitory effect on the viability of mouse hippocampal neuronal HT22 cells compared with that on C6 cells. Additionally, in contrast to PTX alone, PTX-Tf-NPs treatment of C6 cells at lower concentrations (0.0032, 0.0160 and $0.0800 \mu \mathrm{g} / \mathrm{ml}$ ) induced increased $\mathrm{G}_{2} / \mathrm{M}$ arrest, although this difference did not occur at a higher drug concentration $(0.4 \mu \mathrm{g} / \mathrm{ml})$. It was observed that FITC-labeled PTX-Tf-NPs were endocytosed by C6 cells within $4 \mathrm{~h}$. Furthermore, FITC-labeled PTX-Tf-NPs or Tf-NPs co-localized with a lysosomal tracker, Lysotracker Red DND-99. These results of the present study indicated that Tf-NPs enhanced the cytotoxicity of PTX in glioblastoma C6 cells, suggesting that PTX-Tf-NPs should be further explored in animal models of glioblastoma.

\section{Introduction}

Glioblastoma is the most common primary malignant tumor in the central nervous system (CNS), accounting for $46.1 \%$ of the total malignant tumors in the United States that are diagnosed in the brain and spinal cord (1-3). Glioblastoma has been indicated to constitute the majority $(55.1 \%)$ of gliomas that originate from glial cells or precursor cells $(1,4)$ and to be highly aggressive, which is reflected by its classification as a grade IV neoplasm in CNS tumors (5). The incidence of glioblastoma has been estimated to be 3.2 per 100,000 individuals worldwide (1). Current treatment strategies for glioblastoma include the combination of surgical resection with radiotherapy, systemic chemotherapy and local treatment with carmustine wafer, immunotherapy with the anti-angiogenic drug bevacizumab and electric field-based treatment (6-10). However, patients with glioblastoma undergoing the aforementioned treatments have been indicated to exhibit a poor prognosis with a 5-year survival rate of $\sim 5 \%$ and a median survival of $\sim 15$ months $(1,11,12)$. The majority of patients has been revealed to ultimately succumb to the disease due to local recurrence of the tumor (13), which results from the infiltrative viability of glioblastoma that often hinders the complete surgical removal of the initial tumors. Additionally, 
the blood-brain barrier (BBB) has been indicated to prevent chemotherapy drugs from entering the brain, and even when the drugs can be successfully delivered to the tumor, glioblastomas have developed mechanisms of drug resistance, which render chemotherapy less effective (8). Therefore, an urgent need still exists to improve current treatment modalities and/or to develop novel therapeutic strategies to ameliorate the clinical outcomes of patients with glioblastoma.

Strategies focused on enhancing the delivery of cytotoxic drugs or bioreactive agents into the glioblastoma tumor cells with nanocarriers have been previously implemented in preclinical studies and clinical trials $(8,14-17)$. The efficiency of targeted delivery for nanocarriers is determined by their size, surface charge, surface hydration and targeting moiety (15). An ideal nanocarrier has been indicated to be able to cross the highly selective BBB to reach the infiltrating glioblastoma cells, as well as to extravasate via the disrupted blood-brain tumor barrier to enter into the principal tumor core $(15,18)$. Moreover, the nanocarrier should be able to bypass the multidrug resistance efflux transporters, such as P-glycoprotein (P-gp), the multidrug resistance proteins (MRPs) and breast cancer resistance protein, which are usually overexpressed in glioblastoma cells and contribute to the tumor inherent resistance to multiple chemotherapy drugs (19).

The anticancer drug paclitaxel (PTX) has been indicated to inhibit glioblastoma viability in mice; however, this antitumor effect was revealed to be reduced in endothelial and glioblastoma cells where P-gp was overexpressed, thereby conferring resistance to PTX, and preventing the entrance of PTX into tumor cells $(20,21)$. Therefore, the development of a potent carrier to deliver PTX into glioblastoma cells is required. The present study investigated whether previously reported nanoparticle (20) may enhance the antitumor activity of PTX in rat glioblastoma C6 cells in vitro. This nanoparticle is composed of amphiphilic poly ( $\gamma$-glutamic acid-maleimideco-L-lactide)-1,2-dipalmitoylsn-glycero-3-phosphoethanolamine ( $\gamma$-PGA-MAL-PLA-DPPE) copolymer conjugated with a targeting moiety transferrin (Tf) and exhibits a great potential to be an ideal nanocarrier (20). To address these issues, the present study aimed to develop an efficient nano-delivery system for delivering therapeutic agents into glioblastoma cells. The present study provided solid evidence for subsequent investigation of PTX-Tf-NPs in animal models of glioblastoma to better elucidate the antitumor potency of PTX-Tf-NPs.

\section{Materials and methods}

MTT assay. Transferrin (Tf), 4-Nitro-phenyl chloroformate (pNP) (97\%), and paclitaxel (PTX) were obtained from Alfa Aesar, Sigma-Aldrich (Merck KGaA) and Beijing HuaFeng Unite Co. Ltd., respectively, and were prepared as previously described (22). MTT assay was used to evaluate the effect of Tf-NPs, PTX and PTX-Tf-NPs on cell viability in C6 (glioblastoma) and HT22 cells (mouse hippocampal neuron cell line, purchased from American Type Culture Collection).

Cell culture. The cells were cultured in DMEM (Thermo Fisher Scientific, Inc.) supplemented with $10 \%$ FBS (Thermo Fisher Scientific, Inc.) and $1.2 \%$ penicillin/streptomycin for $24-48 \mathrm{~h}$ in a humidified $5 \% \mathrm{CO}_{2}$ atmosphere at $37^{\circ} \mathrm{C}$. Cells at the logarithmic growth phase were washed twice with PBS, trypsinized at $37^{\circ} \mathrm{C}$ incubator for $5 \mathrm{~min}$ and counted using a hemocytometer. A total of $1 \times 10^{4}$ cells were seeded into each well of a 96-well plate. After $24 \mathrm{~h}$, the culture media were removed, and $100 \mu \mathrm{l}$ DMEM containing Tf-NPs at different concentrations $(10.0000,2.0000,0.4000,0.0800,0.0160$ or $0.0032 \mu \mathrm{g} / \mathrm{ml}$ ) were added into the cells in quadruplicate. The same concentrations of PTX or PTX-Tf-NPs were also assayed on the cultured cells in quadruplicate. Cells without treatment served as a blank control. After $48 \mathrm{~h}$, the culture media containing Tf-NPs, PTX or PTX-Tf-NPs were removed, and the cells were washed with PBS. A standard MTT assay was subsequently performed to measure the cytotoxicity of the drugs. The purple formazan was dissolved in DMSO and the absorbance was measured at $570 \mathrm{~nm}$. The experiment was repeated three times. The cell viability curves with different concentrations of Tf-NPs, PTX or PTX-Tf-NPs were plotted.

Flow cytometry. Flow cytometry was performed to analyze the cell cycle distribution of C6 and HT22 cells treated with PTX or PTX-Tf-NPs. C6 or HT22 cells at the logarithmic growth phase were trypsinized at $37^{\circ} \mathrm{C}$ incubator for $5 \mathrm{~min}$ and counted with a hemocytometer. A total of $2 \times 10^{5}$ cells were seeded into each well of a 6 -well plate. When the cells entered the logarithmic growth phase after $24 \mathrm{~h}$, they were treated with PTX, PTX-Tf-NPs (dissolved in cell culture media) at the concentrations of $0.0032,0.016,0.080$ or $0.400 \mu \mathrm{g} / \mathrm{ml}$ (for C6 cells) or $0.06,0.25$ or $1.00 \mu \mathrm{g} / \mathrm{ml}$ (for HT22 cells) in duplicate. Cell culture medium was used as a solvent control. After $48 \mathrm{~h}$, the cells were collected and centrifuged at $705 \mathrm{x} \mathrm{g}$ at $4^{\circ} \mathrm{C}$ for $5 \mathrm{~min}$. The cells were washed twice with pre-cooled PBS and fixed with pre-cooled $70 \%$ ethanol at $4^{\circ} \mathrm{C}$ for $30 \mathrm{~min}$. Following fixation, the cells were centrifuged at $705 \mathrm{x} g$ for $5 \mathrm{~min}$, and $70 \%$ ethanol was removed. RNA was digested with $100 \mu \mathrm{l}$ of RNase A $(0.1 \mathrm{mg} / \mathrm{ml})$ at $37^{\circ} \mathrm{C}$ for $30 \mathrm{~min}$. The cells were washed twice with PBS and subsequently stained with $200 \mu \mathrm{l}$ propidium iodide (PI, Beyotime Institute of Biotechnology) $(0.05 \mathrm{mg} / \mathrm{ml})$ containing $0.03 \%$ Triton X-100 at $4^{\circ} \mathrm{C}$ for $30 \mathrm{~min}$. The cell suspension was filtered using a nylon mesh with $40 \mu \mathrm{m}$ pores before being analyzed using a FACSAria flow cytometer (Becton, Dickinson and Company) and analyzed using FlowJo 7.6 (FlowJo LLC). The percentages of cells at $G_{0} / G_{1}, S$ and $G_{2} / M$ phases were calculated. The experiment was repeated three times.

Examination of the uptake and subcellular localization of nanoparticles. A total of $4 \times 10^{5} \mathrm{C} 6$ cells were seeded in a $35 \mathrm{~mm}$ dish with an integrated cover glass bottom of a $0.1 \mathrm{~mm}$ thickness and $14 \mathrm{~mm}$ diameter (MatTek Corporation). After $24 \mathrm{~h}$, the cells were washed twice with PBS and incubated at $37^{\circ} \mathrm{C}$ with DMEM containing $10 \mu \mathrm{g} / \mathrm{ml}$ FITC-conjugated PTX-Tf-NPs for $0,0.5,1,2$ and $4 \mathrm{~h}$. The cells were washed with PBS before being examined under FLUOVIEW FV1000 confocal laser scanning microscope (magnification, $\mathrm{x} 40$; Olympus Corporation).

C6 cells were stained with 75 nM LysoTracker $\operatorname{Red}^{\mathrm{TM}}$ DND-99 at $37^{\circ} \mathrm{C}$ for $1 \mathrm{~h}$ (Invitrogen; Thermo Fisher Scientific, Inc.) following incubation at $37^{\circ} \mathrm{C}$ with $10 \mu \mathrm{g} / \mathrm{ml}$ FITC-conjugated PTX-Tf-NPs or Tf-NPs for $4 \mathrm{~h}$. The cells were washed three times with Dulbecco's PBS before being 
Table I. Cell viability of C6 cells following treatment with Tf-NPs, PTX or PTX-Tf-NPs.

Concentration $(\mu \mathrm{g} / \mathrm{ml})$

\begin{tabular}{lrrrrrr}
\cline { 2 - 6 } Drug & \multicolumn{1}{c}{0.0032} & \multicolumn{1}{c}{0.0160} & 0.0800 & 0.4000 & 2.0000 & 10.0000 \\
\hline Tf-NPs & $119.11 \pm 2.45$ & $103.01 \pm 3.07$ & $91.89 \pm 1.52$ & $96.69 \pm 1.63$ & $96.64 \pm 0.85$ & $87.89 \pm 2.44$ \\
PTX & $91.19 \pm 1.94$ & $87.21 \pm 3.13$ & $83.12 \pm 1.67$ & $81.19 \pm 1.91$ & $74.28 \pm 2.81$ & $62.14 \pm 0.07$ \\
PTX-Tf-NPs & $94.66 \pm 0.81$ & $91.4 \pm 2.12$ & $83.09 \pm 0.44$ & $78.0 \pm 1.23^{\mathrm{a}}$ & $68.6 \pm 1.12^{\mathrm{a}}$ & $56.25 \pm 0.20^{\mathrm{a}}$
\end{tabular}

${ }^{\mathrm{a}} \mathrm{P}<0.01$ vs. PTX. PTX, paclitaxel; Tf-NPs, transferrin-nanoparticles.

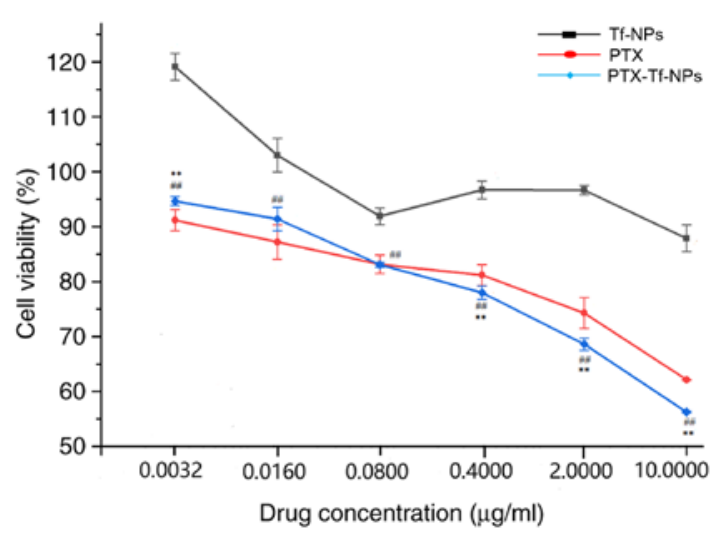

Figure 1. PTX or PTX-Tf-NP treatment reduces the viability of rat glioblastoma C6 cells in a dose-dependent manner, but PTX-Tf-NPs exhibit a stronger inhibitory effect at higher concentrations. C6 cells were treated with Tf-NPs, PTX or PTX-Tf-NPs at the concentrations of $0.0032,0.016,0.08$, $0.4,2$ or $10 \mu \mathrm{g} / \mathrm{ml}$ for $48 \mathrm{~h}$, followed by detection of the cell viability using an MTT assay. The data are presented as the mean $\pm \mathrm{SD}$, and ANOVA followed by Tukey's post hoc test was used for statistical analysis. ${ }^{* *} \mathrm{P}<0.01$ vs. PTX; ${ }^{\# \#} \mathrm{P}<0.01$ vs. Tf-NPs. PTX, paclitaxel; Tf-NPs, transferrin-nanoparticles.

examined under FLUOVIEW FV1000 confocal laser scanning microscope (magnification, x40; FV10 ASW, Olympus Corporation). The filter sets for FITC and LysoTracker Red DND-99 were $488 \mathrm{~nm}$ (excitation)/510 nm (emission) and $488 \mathrm{~nm}$ (excitation)/560 nm (emission), respectively.

Statistical analysis. The experiments were repeated three times. The data are presented as the mean \pm SD. SPSS 13.0 software (SPSS, Inc.) was used for data analysis. One-way ANOVA followed by Turkey's post hoc test was used for the comparison of multiple groups in Figs. 1 and 2 . $\mathrm{P}<0.05$ was considered to indicate a statistically significant difference.

\section{Results}

PTX or PTX-Tf-NPs reduce the viability of rat glioblastoma C6 cells in a dose-dependent manner, but PTX-Tf-NPs exhibit a stronger inhibitory effect at higher concentrations compared with PTX. Rat glioblastoma C6 cells were treated with Tf-NPs, PTX or PTX-Tf-NPs for $48 \mathrm{~h}$, and cell viability was detected using the MTT assay. The percentages of cell viability are presented in Fig. 1 and Table I. The results indicated that treatment with Tf-NPs at concentrations of 0.0032 or $0.016 \mu \mathrm{g} / \mathrm{ml}$ did not inhibit C6 cell viability, whereas Tf-NP treatment at concentrations of $0.08,0.4,2$ and $10 \mu \mathrm{g} / \mathrm{ml}$ resulted in a

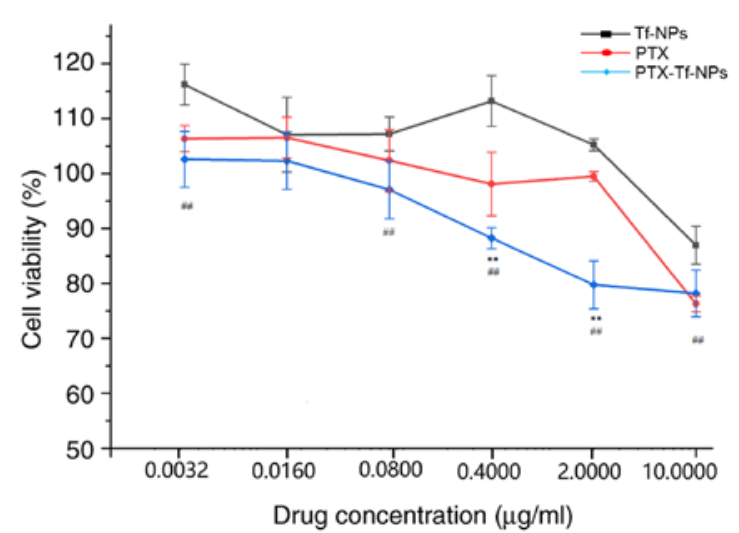

Figure 2. PTX and PTX-Tf-NPs exhibit a lower inhibitory effect on the cell viability of mouse hippocampal neuronal HT22 cells compared with that on rat glioblastoma C6 cells. C6 cells were treated with Tf-NPs, PTX or PTX-Tf-NPs at the concentrations of $0.0032,0.016,0.08,0.4,2$ or $10 \mu \mathrm{g} / \mathrm{ml}$ for $48 \mathrm{~h}$, followed by detection of the cell viability using an MTT assay. The data are presented as the mean $\pm \mathrm{SD}$, and ANOVA followed by Tukey's post hoc test was used for statistical analysis. ${ }^{* *} \mathrm{P}<0.01$ vs. $\mathrm{PTX} ;{ }^{\# \#} \mathrm{P}<0.01$ vs. Tf-NPs. PTX, paclitaxel; Tf-NPs, transferrin-nanoparticles.

cell viability of 92,97, 97 and $88 \%$ in C6 cells, respectively, compared with control cells, indicating that Tf-NPs alone cause a low cytotoxicity in C6 cells. Both PTX and PTX-Tf-NPs exhibited a dose-dependent effect on cell viability in C6 cells. Following PTX treatment at concentrations of 0.0032, 0.016 and $0.08 \mu \mathrm{g} / \mathrm{ml}$, C6 cell viability was 91,87 and $83 \%$, respectively, while following PTX-Tf-NP treatment, cell viability was 95, 91 and $83 \%$, respectively. Statistical analysis revealed that at a concentration of $\leq 0.08 \mu \mathrm{g} / \mathrm{ml}$, no significant difference in cell viability by treatment with either PTX or PTX-Tf-NPs was observed, indicating that PTX and PTX-Tf-NPs exhibit similar cell viability inhibitory effects at these concentrations. Nevertheless, at concentrations of $0.4,2$ and $10 \mu \mathrm{g} / \mathrm{ml}$, C6 cells treated with PTX exhibited an average viability of 81,74 and $62 \%$, respectively, but C6 cells treated with PTX-Tf-NPs exhibited significantly lower viability compared with cells treated with PTX (78, 69 and 56\%, respectively). This suggested that PTX-Tf-NPs were more potent compared with PTX in reducing the viability of C6 glioblastoma cells at higher concentrations.

PTX and PTX-Tf-NPs exhibit a lower inhibitory effect on the viability of mouse hippocampal neuronal HT22 cells compared with that on rat glioblastoma C6 cells. Immortalized mouse hippocampal neuronal HT22 cells were treated with Tf-NPs, 
Table II. Cell viability of HT22 cells following treatment with Tf-NPs, PTX or PTX-Tf-NPs.

\begin{tabular}{|c|c|c|c|c|c|c|}
\hline \multirow[b]{2}{*}{ Drug } & \multicolumn{6}{|c|}{ Concentration $(\mu \mathrm{g} / \mathrm{ml})$} \\
\hline & 0.0032 & 0.0160 & 0.0800 & 0.4000 & 2.0000 & 10.0000 \\
\hline Tf-NPs & $116.2 \pm 3.70$ & $107.05 \pm 6.80$ & $107.2 \pm 3.09$ & $113.19 \pm 4.63$ & $105.24 \pm 1.08$ & $86.96 \pm 3.46$ \\
\hline PTX & $106.35 \pm 2.35$ & $106.53 \pm 3.70$ & $102.36 \pm 5.66$ & $98.1 \pm 5.77$ & $99.47 \pm 0.88$ & $76.27 \pm 1.43$ \\
\hline Tf-NPs-PTX & $102.57 \pm 5.10$ & $102.32 \pm 5.23$ & $97.07 \pm 5.28$ & $88.25 \pm 1.90^{\mathrm{a}}$ & $79.74 \pm 4.35^{\mathrm{a}}$ & $78.19 \pm 4.26^{\mathrm{a}}$ \\
\hline
\end{tabular}

${ }^{\mathrm{a} P}<0.01$ vs. PTX. PTX, paclitaxel; Tf-NPs, transferrin-nanoparticles.
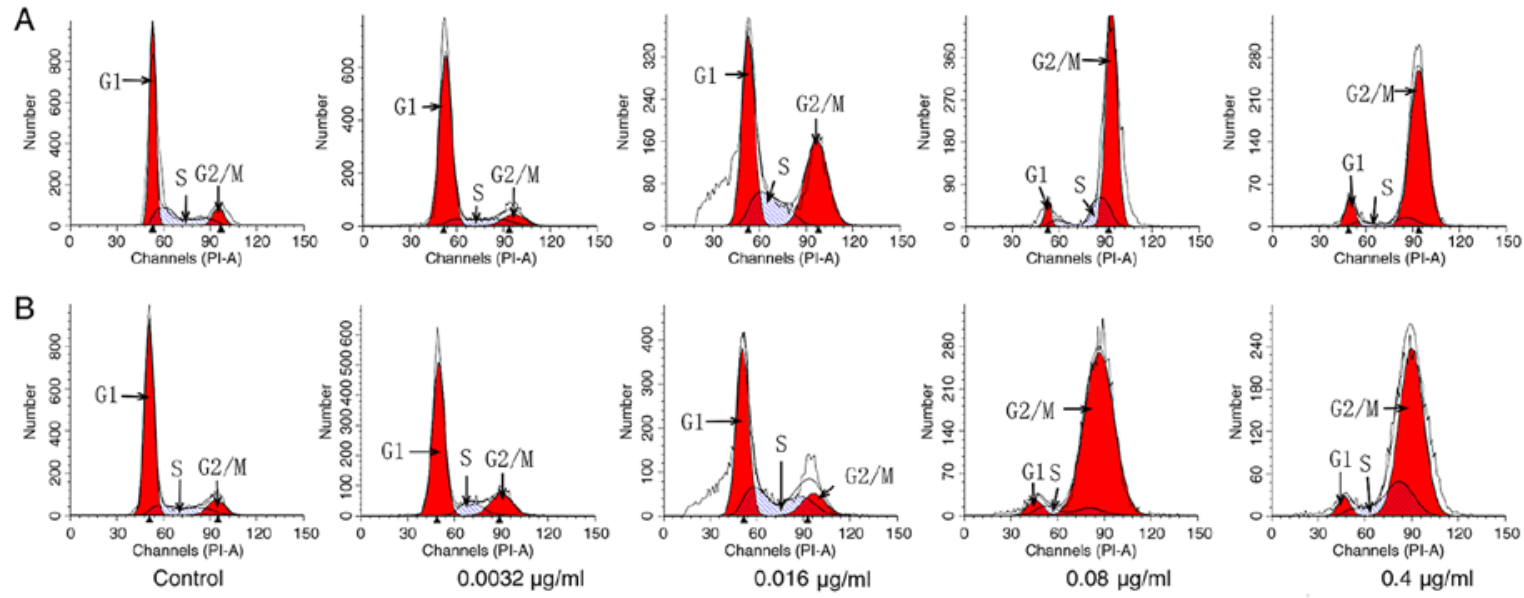

Figure 3. PTX-Tf-NP and PTX treatment induces $\mathrm{G}_{2} / \mathrm{M}$ cell cycle arrest differentially in C6 cells. C6 cells were treated with PTX or PTX-Tf-NPs at the concentrations of $0.0032,0.016,0.08$ or $0.4 \mu \mathrm{g} / \mathrm{ml}$ for $48 \mathrm{~h}$. The cells were stained with PI solution and were subsequently analyzed by flow cytometry. (A) PTX and (B) PTX-Tf-NPs. PTX, paclitaxel; Tf-NPs, transferrin-nanoparticles; PI, propidium iodide.

PTX or PTX-Tf-NPs for $48 \mathrm{~h}$, and cell viability was measured using an MTT assay. The cell viability measurements are presented in Fig. 2 and Table II. The results indicated that at a high concentration of $10 \mu \mathrm{g} / \mathrm{ml}$, Tf-NP treatment reduced HT22 cell viability to $87 \%$. At a concentration of $\leq 2 \mu \mathrm{g} / \mathrm{ml}$, Tf-NPs did not affect HT22 cell viability. These data indicated that treatment with Tf-NPs alone results in low cytotoxicity in HT22 cells. Interestingly, PTX alone did not inhibit cell viability at a concentration of $\leq 2 \mu \mathrm{g} / \mathrm{ml}$, but reduced HT22 cell viability to $76 \%$ relatively to that of control cells at a concentration of $10 \mu \mathrm{g} / \mathrm{ml}$ (Fig. 2), which was different from that in C6 cells (Fig. 1). PTX-Tf-NPs did not inhibit HT22 cell viability at a concentration of $\leq 0.08 \mu \mathrm{g} / \mathrm{ml}$, but reduced HT22 cell viability to 88,80 and $78 \%$ relatively to that of control cells at the concentrations of $0.4,2.0$ and $10 \mu \mathrm{g} / \mathrm{ml}$, respectively (Fig. 2). These data indicated that PTX-Tf-NPs exhibited a lower inhibitory effect on the viability of HT22 cells compared with that on C6 cells.

PTX and PTX-Tf-NPs induce $G_{2} / M$ arrest differentially in C6 cells. Following PTX treatment at the concentrations of $0.0032,0.016,0.08$ or $0.4 \mu \mathrm{g} / \mathrm{ml}$, C6 cells exhibited 9, 28, 73 and $86 \% \mathrm{G}_{2} / \mathrm{M}$ distribution, respectively (Fig. 3A and Table III), whereas untreated cells presented only $9 \%$. These data suggested that PTX treatment at the concentrations of $0.016,0.08$ and $0.4 \mu \mathrm{g} / \mathrm{ml}$ resulted in $\mathrm{G}_{2} / \mathrm{M}$ arrest in C6 cells, and the percentage of $\mathrm{G}_{2} / \mathrm{M}$ arrest was associated with the concentration of PTX. On the other hand, following PTX-Tf-NP treatment at the concentrations of $0.0032,0.016,0.08$ and $0.4 \mu \mathrm{g} / \mathrm{ml}$, C6 cells exhibited $15,17,89$ and $71 \% \mathrm{G}_{2} / \mathrm{M}$ distribution, respectively (Fig. 3B and Table III). These data indicated that PTX-Tf-NPs induced $\mathrm{G}_{2} / \mathrm{M}$ cell cycle arrest in $\mathrm{C} 6$ cells even at the lowest concentration of $0.0032 \mu \mathrm{g} / \mathrm{ml}$. However, the $\mathrm{G}_{2} / \mathrm{M}$ arrest effect of PTX-Tf-NPs peaked at a concentration of $0.08 \mu \mathrm{g} / \mathrm{ml}$, and this effect was reduced at a concentration of $0.4 \mu \mathrm{g} / \mathrm{ml}$. In addition, the aforementioned results demonstrated that at the concentrations of 0.0032 and $0.08 \mu \mathrm{g} / \mathrm{ml}$, PTX-Tf-NPs resulted in a higher percentage of $\mathrm{G}_{2} / \mathrm{M}$ arrest compared with PTX, but this difference was reversed at the concentration of $0.4 \mu \mathrm{g} / \mathrm{ml}$.

PTX and PTX-Tf-NPs induce $G_{2} / M$ arrest differentially in HT22 cells. Following PTX treatment at the concentrations of $0.06,0.25$ or $1 \mu \mathrm{g} / \mathrm{ml}, \mathrm{HT} 22$ cells exhibited 20,52 and $73 \% \mathrm{G}_{2} / \mathrm{M}$ distribution, respectively, whereas the untreated cells exhibited only $14 \%$ (Fig. 4, row 1; Table IV), indicating that $\mathrm{G}_{2} / \mathrm{M}$ cell cycle arrest in HT22 cells was associated with the concentration of PTX. On the contrary, HT22 cells treated with $0.06,0.25$ or $1 \mu \mathrm{g} / \mathrm{ml}$ PTX-Tf-NPs exhibited 4, 29 or $66 \% \mathrm{G}_{2} / \mathrm{M}$ phase distribution, respectively (Fig. 4, row 2; Table IV). These data suggested that PTX-Tf-NPs induced $\mathrm{G}_{2} / \mathrm{M}$ cell cycle arrest in HT22 cells at the concentrations of 0.25 and $1 \mu \mathrm{g} / \mathrm{ml}$, but not $0.06 \mu \mathrm{g} / \mathrm{ml}$. Moreover, at these concentrations, PTX-Tf-NPs resulted in a lower percentage of $\mathrm{G}_{2} / \mathrm{M}$ arrest compared with PTX. 
Table III. Cell cycle analysis of C6 cells following treatment with PTX or PTX-Tf-NPs.

\begin{tabular}{lcccc}
\hline Treatment & Concentration $(\mu \mathrm{g} / \mathrm{ml})$ & $\mathrm{G}_{0} / \mathrm{G}_{1}$ phase $(\%)$ & S phase $(\%)$ & $\mathrm{G}_{2} / \mathrm{M}_{\mathrm{phase}}(\%)$ \\
\hline Control & - & $62.26 \pm 1.68$ & $28.28 \pm 2.21$ & \\
PTX & 0.0032 & & & \\
& 0.0160 & $68.07 \pm 8.19$ & $22.49 \pm 8.61$ & $28.45 \pm 0.41$ \\
& 0.0800 & $49.08 \pm 5.38$ & $22.59 \pm 2.35$ & $73.42 \pm 1.97$ \\
& 0.4000 & $5.57 \pm 0.44$ & $21.01 \pm 1.53$ & $86.3 \pm 4.02$ \\
PTX-Tf-NPs & 0.0032 & $7.14 \pm 1.30$ & $6.57 \pm 2.70$ & $14.58 \pm 3.84^{\mathrm{a}}$ \\
& 0.0160 & $70.68 \pm 1.46^{\mathrm{a}}$ & $25.7 \pm 6.42$ & $16.58 \pm 2.70^{\mathrm{a}}$ \\
& 0.0800 & $57.73 \pm 3.70^{\mathrm{a}}$ & $5.77 \pm 1.88$ & $88.61 \pm 0.66^{\mathrm{a}}$ \\
& 0.4000 & $5.62 \pm 1.22^{\mathrm{a}}$ & $23.68 \pm 2.23$ & $70.83 \pm 3.36^{\mathrm{a}}$ \\
\hline
\end{tabular}

${ }^{\mathrm{a} P}<0.01$ vs. PTX. PTX, paclitaxel; Tf-NPs, transferrin-nanoparticles.

Table IV. Cell cycle analysis of HT22 cells.

\begin{tabular}{lcccc}
\hline Treatment & Concentration $(\mu \mathrm{g} / \mathrm{ml})$ & $\mathrm{G}_{0} / \mathrm{G}_{1}$ phase $(\%)$ & S phase $(\%)$ & $\mathrm{G}_{2} / \mathrm{M}_{\mathrm{phase}}(\%)$ \\
\hline Control & - & $40.83 \pm 2.76$ & $45.03 \pm 3.71$ & $14.14 \pm 0.56$ \\
PTX & 0.06 & $29.52 \pm 3.06$ & $50.85 \pm 3.54$ & $19.64 \pm 0.48$ \\
& 0.25 & $7.89 \pm 0.78$ & $40.39 \pm 0.15$ & $72.61 \pm 4.92$ \\
PTX-Tf-NPs & 1.00 & $8.86 \pm 2.39$ & $18.54 \pm 2.53$ & $4.46 \pm 3.38^{\mathrm{a}}$ \\
& 0.06 & $39.38 \pm 0.08^{\mathrm{a}}$ & $56.17 \pm 3.46$ & $28.68 \pm 3.00^{\mathrm{a}}$ \\
& 0.25 & $12.82 \pm 2.19^{\mathrm{a}}$ & $58.5 \pm 5.41$ & $66.36 \pm 5.52^{\mathrm{a}}$ \\
\hline
\end{tabular}

${ }^{\mathrm{a}} \mathrm{P}<0.01$ vs. PTX. PTX, paclitaxel; Tf-NPs, transferrin-nanoparticles.
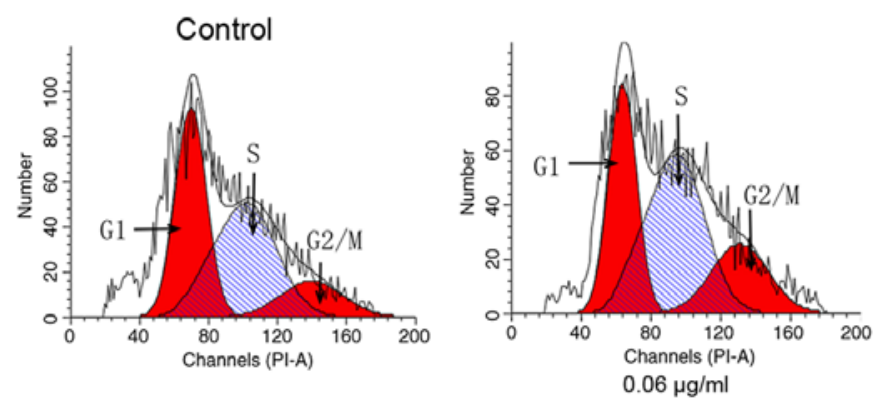

Control

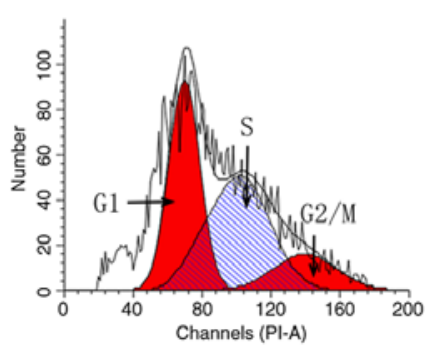

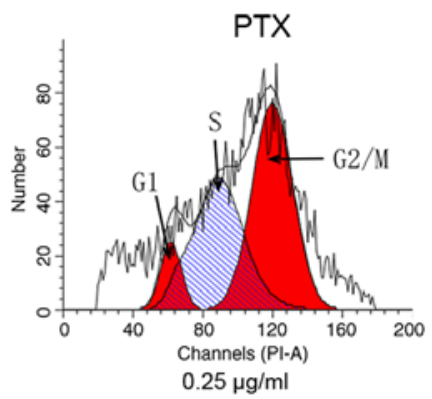

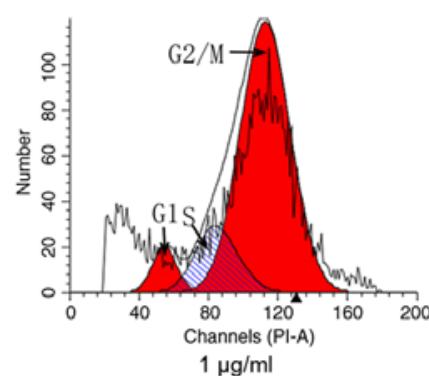

PTX-Tf-NPs
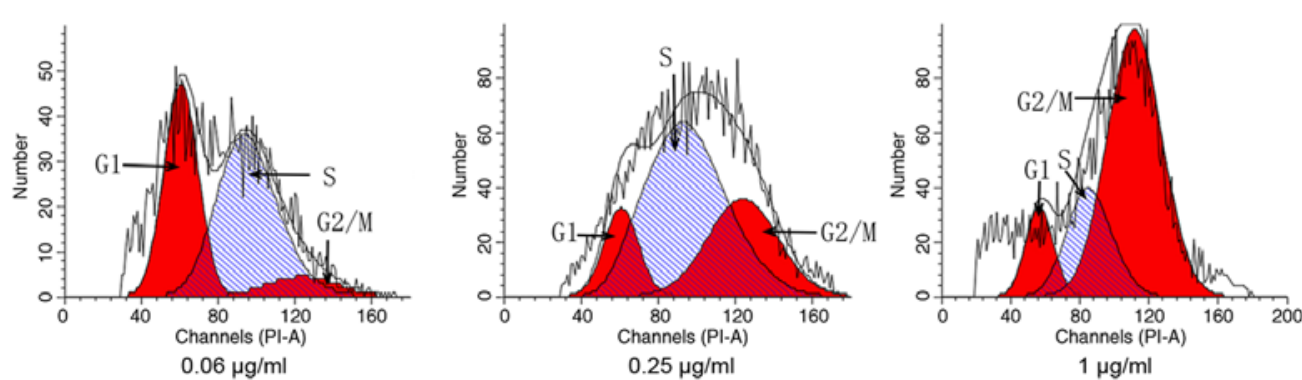

Figure 4. Cell cycle analysis of HT22 cells. HT22 cells were treated with PTX or PTX-Tf-NPs at the concentrations of $0.06,0.25 \mathrm{or} 1.0 \mu \mathrm{g} / \mathrm{ml}$ for $48 \mathrm{~h}$. The cells were subsequently stained with PI solution and analyzed by flow cytometry. Control: Row 1, 1st panel; PTX: Row 1, 2nd-4th panels; Control: Row 2, 1st panel; PTX-Tf-NPs: Row 2, 2nd-4th panels. PTX, paclitaxel; Tf-NPs, transferrin-nanoparticles; Control, without drug treatment; PI, propidium iodide. 


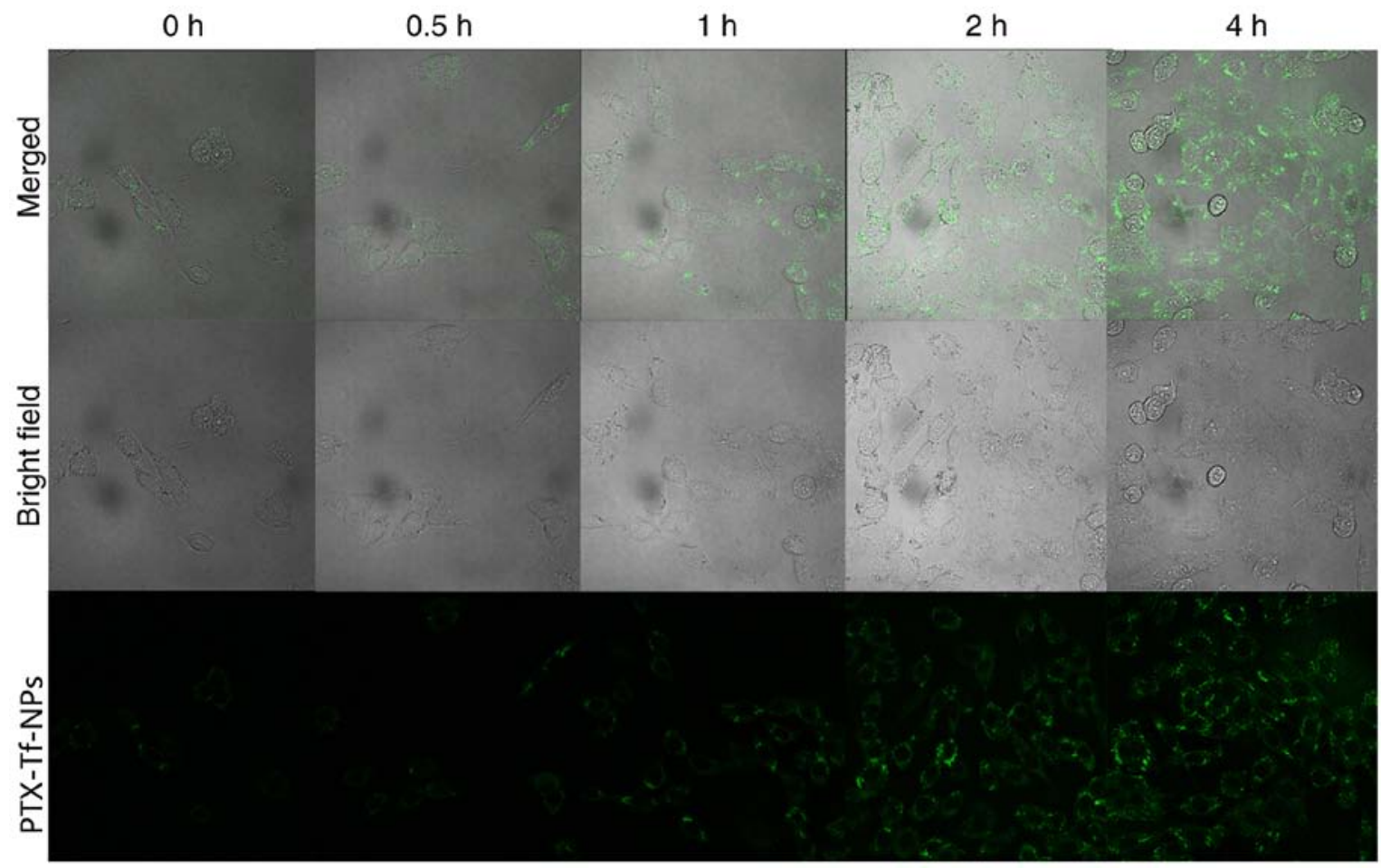

Figure 5. Endocytosis of PTX-Tf-NPs in C6 cells increases in a time-dependent manner. The cells were treated with $10 \mu \mathrm{g} / \mathrm{ml}$ FITC-labeled PTX-Tf-NPs for $0,0.5,1,2$ and $4 \mathrm{~h}$. Green fluorescence was examined using confocal laser microscopy. The left column includes fluorescent images (magnification, x 40 ) of FITC-labeled PTX-Tf-NPs. Images in the middle column were captured under bright field (magnification, $\mathrm{x} 40$ ), and the merged images of the left and middle columns are presented in the right column. PTX-Tf-NPs, paclitaxel-transferrin-nanoparticles.

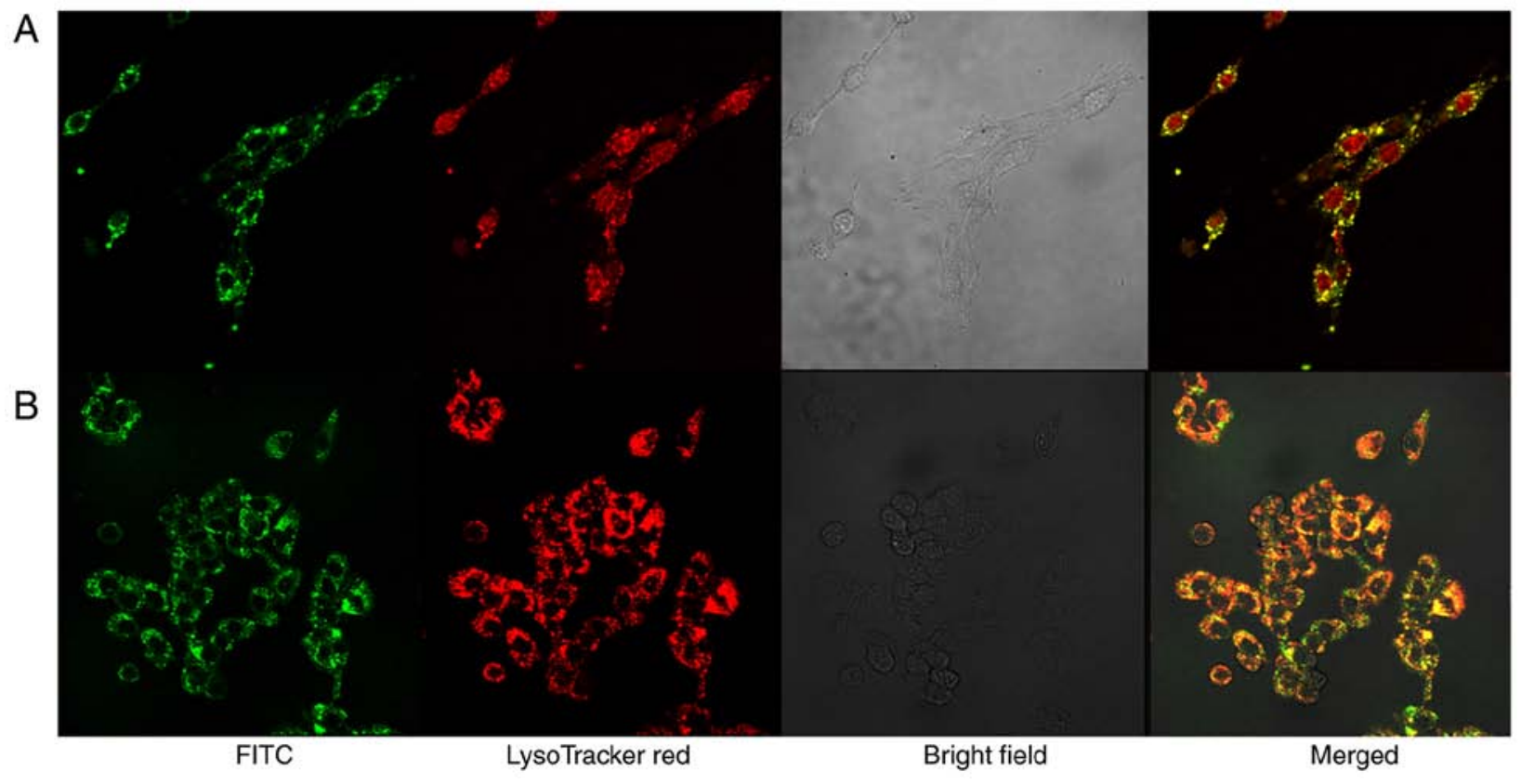

Figure 6. PTX-Tf-NPs or TF-NPs are co-localized with lysosomes. C6 cells were treated with $10 \mu \mathrm{g} / \mathrm{ml}$ FITC-labeled PTX-Tf-NPs or TF-NPs for 4 h, and subsequently incubated with $75 \mathrm{nM}$ LysoTracker Red DND-99 for an additional $1 \mathrm{~h}$. The fluorescence was examined using a confocal laser microscope (magnification, x40). (A) Tf-NPs and (B) PTX-Tf-NPs. From left to right, FITC-labeled PTX-Tf-NPs or TF-NPs, LysoTracker Red DND-99, bright field and merged images of the other three columns are presented. PTX, paclitaxel; Tf-NPs, transferrin-nanoparticles.

PTX-Tf-NP endocytosis in C6 cells increases in a time-dependent manner. To visualize the distribution of PTX-Tf-NPs in C6 cells, a high concentration of the particles $(10 \mu \mathrm{g} / \mathrm{ml})$ was used, as at this concentration an increased amount of particles is more likely to enter the cells. The cells were incubated with
FITC-labeled PTX-Tf-NPs and the green fluorescence signal was detected under confocal laser microscope. Following treatment for $0.5 \mathrm{~h}$, a small amount of fluorescence signal was detected in the cytosol of $\mathrm{C} 6$ cells, indicating that PTX-Tf-NPs had entered into C6 cells (Fig. 5). With increased incubation 
time, an increased number of PTX-Tf-NPs entered C6 cells. Following treatment for $4 \mathrm{~h}$, a strong fluorescence signal was visible in nearly all C6 cells (Fig. 5), indicating that the majority of PTX-Tf-NPs were successfully endocytosed by C6 cells.

Intracellular PTX-Tf-NPs or Tf-NPs are co-localized with lysosomes. C6 cells were treated with FITC-labeled PTX-Tf-NPs or Tf-NPs and additionally incubated with LysoTracker Red DND-99. LysoTracker Red DND-99 can specifically accumulate in acidic organelles, such as lysosomes (21). The fluorescence signals were examined using a confocal laser microscope. As demonstrated in Fig. 6, FITC-labeled PTX-Tf-NPs or Tf-NPs co-localized with LysoTracker Red DND-99. These data suggested that both PTX-Tf-NPs and TF-NPs are localized in lysosomes upon endocytosis.

\section{Discussion}

The present study demonstrated that PTX-Tf-NP treatment resulted in a higher cytotoxicity in glioblastoma C6 cells at the higher concentrations tested $(0.4,2$ and $10 \mu \mathrm{g} / \mathrm{ml})$ compared with PTX alone. Additionally, it was observed that PTX-Tf-NPs induced higher $\mathrm{G}_{2} / \mathrm{M}$ arrest at the lower concentrations tested $(0.0032$ and $0.0800 \mu \mathrm{g} / \mathrm{ml})$ in C6 cells compared with PTX alone. Moreover, PTX-Tf-NP treatment resulted in a higher cytotoxicity in C6 cells compared with that on mouse hippocampal neuronal HT22 cells. Furthermore, Tf-NPs alone exhibited a low cytotoxicity in both glioblastoma C6 and hippocampal neuronal HT22 cells, only slightly inhibiting the viability of these cells at a high concentration of $10 \mu \mathrm{g} / \mathrm{ml}$, which indicated that Tf-NPs can be safe for therapeutic applications. Therefore, the results of the present study suggested that Tf-NPs exhibit a great potential to be an excellent nanocarrier for glioblastoma drug targeting. Based on the Tf-NP structure, there are two key features that may contribute to the successful targeting of glioblastoma cells by PTX-Tf-NPs in vivo, including the targeting moiety $\mathrm{Tf}$ (22) and the backbone of Tf-NPs, which is composed of an amphiphilic $\gamma$-PGA-MAL-PLA-DPPE copolymer (20).

Under normal physiological conditions, Tf functions as an iron carrier in plasma and transfers iron to various types of tissues, such as the brain (23). Currently, how iron molecules cross the BBB remains unclear. The models of Tf receptor (TfR)-mediated endocytosis and transcytosis have been proposed to demonstrate the movement of iron from endothelial cells in the BBB to the brain parenchyma. Generally, iron-loaded holo-Tf has been indicated to bind to TfR, which is localized on the luminal cell surface of endothelial cells in the brain (24). The complex of holo-Tf with TfR is internalized into the cell via the clathrin-coated pit and wrapped into the clathrin-coated vesicle (24). Subsequently, the vesicle becomes uncoated and fuses with the early endosome. The iron transport via TfR-mediated endocytosis or transcytosis has been revealed to depend on whether holo-Tf can be transferred to brain parenchyma, which is dependent upon the ability of Tf to cross the abluminal membrane together with iron (24). In TfR-mediated endocytosis, the acidified environment inside the endosome has been reported to induce the separation of iron from holo-Tf, and iron has been indicated to be subsequently pumped out of the endosome by divalent metal transporter 1 into the cytosol, followed by the transportation of iron to the abluminal membrane of endothelial cells by ferroportin (24). The majority of TfR andiron-free apo-Tf complexes recycle back to the respective cell surface and plasma, whereas only a small amount of the TfR-Tf complex undergoes lysosomal degradation (25). On the other hand, a certain amount of iron in the form of holo-Tf has been indicated to be transported as a complex across the abluminal membrane of the brain's endothelial cells to reach the brain parenchyma in TfR-mediated transcytosis (24).

As a targeting moiety, Tf may serve vital roles in the transportation of PTX-Tf-NPs across the BBB. TfR1 is highly expressed in endothelial cells from the BBB (26), which makes it a feasible target for the delivery of therapeutic agents into the brain parenchyma via TfR-mediated transcytosis. Several groups have reported that Tf-conjugated nanoparticles enter the brain in greater amounts in vivo compared with those without a Tf targeting moiety (25,27-29). Sonali et al (29) demonstrated that Tf-conjugated gold nanoparticles crossed the BBB in a manner dependent on the avidity of the nanoparticles to TfR, which was determined by the size of the nanoparticles and the amount of Tf conjugated to the surface of the nanoparticles. For example, nanoparticles with a diameter of $45 \mathrm{~nm}$ and $30 \mathrm{Tf} /$ particle have been indicated to reach the mouse brain parenchyma in larger amounts compared with same-sized nanoparticles with either 20 or $100 \mathrm{Tf} /$ particle (29). Nanoparticles with a diameter of $80 \mathrm{~nm}$ and $20 \mathrm{Tf} /$ particle have been reported to enter the mouse brain at a higher number compared with same-sized nanoparticles with $200 \mathrm{Tf} /$ particle (27). Tf-conjugated nanoparticles with a moderate avidity to Tf have been demonstrated to be more likely transcytosed to the brain parenchyma in comparison to nanoparticles with a high avidity to Tf (27). To further increase the amount of transcytosed Tf-conjugated nanoparticles, the same research group incorporated an acid-cleavable linkage between $\mathrm{Tf}$ and the nanoparticle core (28). It has been revealed that this acid-cleavable linkage increased the mobility of high-avidity Tf-containing nanoparticles ( $80 \mathrm{~nm}$ in diameter; $200 \mathrm{Tf} /$ particle), but not low-avidity Tf-containing nanoparticles $(80 \mathrm{~nm}$ in diameter; $20 \mathrm{Tf} /$ particle). High-avidity Tf-containing nanoparticles with the acid-cleavable linkage have been demonstrated to reach the mouse brain parenchyma more efficiently compared with low-avidity Tf-containing nanoparticles without the acid-cleavable linkage (28). This may be attributed to the fact that the Tf-containing nanoparticles with high avidity to TfR may be endocytosed by endothelial cells in the BBB to a greater extent compared with their low-avidity counterparts, whereas the acid-cleavable linkage may induce an endosomal separation of the nanoparticle core from both Tf and TfR and facilitate the ability of the nanoparticle core to enter the transcytotic pathway. However, despite the ability of the acid-cleavable linkage to increase the amount of Tf-containing nanoparticles entering the mouse brain, the transcytosed nanoparticles without the Tf targeting moiety would have lost their targeting ability to glioblastoma cells, which exhibit a high expression of TfR $(30,31)$. 
There are two major advantages for glioblastoma-targeted delivery of drug-loaded Tf-modified nanocarriers via TfR-mediated endocytosis. As aforementioned, one advantage is the achievement of an efficient targeted delivery of anticancer agents via binding of a Tf-modified nanocarrier to TfR, which has been indicated to be upregulated owing to the increased uptake of iron by glioblastoma cells $(32,33)$. Another advantage is bypassing the multi-drug resistance developed by glioblastoma cells due to their increased expression of efflux transporters, such as MRP1 (32). Inhibition of MRP1 or downregulation of MRP1 has been demonstrated to improve the sensitivity of glioblastoma cells to chemotherapy drugs in vitro $(19,33,34)$. By taking into account the advantages of a Tf-modified nanocarrier that may facilitate the transport of anticancer agents into glioblastoma cells, the present study using in vitro methods, as well as previous studies using in vivo methods, demonstrated that PTX-Tf-NPs in addition to other drug-loaded Tf-containing nanoparticles (Tf-PO-DOX, G4-DOX-PEG-Tf-TAM, Tf-NP-DOX, NPs-ZOL-Tf) (35-38) inhibited the viability of glioblastoma cells in comparison to anticancer drugs or a drug-loaded nanocarrier without Tf modification. Liu et al (36) prepared doxorubicin (Dox)-loaded Tf-conjugated polyethylene glycol-polylactic acid NPs (Tf-NP-Dox) and observed a 2 -fold increase in the intracellular drug concentration in C6 cells compared with NP-Dox alone. Pang et al (35) further revealed that Tf-conjugated biodegradable polymersomes (Tf-PO) enhanced the delivery of Dox into the brain and tumors in glioblastoma-bearing rats in comparison to PO alone. The present study for the first time, to the best of our knowledge, demonstrated that drug-loaded Tf-NP treatment resulted in a higher cytotoxicity in glioblastoma cells (C6 cells) compared with normal neural cells (HT22 cells), which supported the use of Tf in specifically targeting glioblastoma cells. Moreover, the current study indicated that PTX-Tf-NPs were localized in the lysosomes upon endocytosis in these glioblastoma cells.

In addition to the Tf targeting moiety, the backbone of Tf-NPs that was used in the present study, which was composed of amphiphilic $\gamma$-PGA-MAL-PLA-DPPE copolymer, has been indicated to exhibit several ideal characteristics for the systemic delivery of anticancer drugs into the brain. Firstly, $\gamma$-PGA-MAL-PLA-DPPE copolymer has been revealed to be highly biodegradable, as polypeptide $\gamma$-PGA was made from carboxy-linked glutamate residues (39), and PLA (40) and phospholipid DPPE (41) have all been reported to exhibit excellent biodegradability and biocompatibility. The results of the present study also indicated that Tf-NPs alone were biocompatible, as they exhibited very low cytotoxicity in C6 and HT22 cells. Secondly, a controlled and sustained release of PTX from PTX-Tf-NPs has been observed at both $\mathrm{pH} 7.4$ and $\mathrm{pH} 5.0$, which simulated the $\mathrm{pH}$ values at physiological conditions and in the lysosomal environment, respectively (20). Lastly, the PTX-Tf-NPs used in the present study have previously been indicated to exhibit desirable near-neutral zeta potentials (20), which may prevent the aggregation of these nanoparticles and their uptake by the mononuclear phagocyte system, thereby achieving a prolonged circulation time in the bloodstream (42).
There is a limitation to the present study. Although it was revealed that the nanoparticles were efficiently transported into cells derived from the $\mathrm{BBB}$, no in vivo evidence was included in the current study to support that these particles can cross the BBB. Additional in vivo assays are required to confirm this effect.

The results of the present study indicated that PTX-Tf-NPs were more potent compared with PTX alone in inhibiting cell viability and inducing $\mathrm{G}_{2} / \mathrm{M}$ cell cycle arrest in rat glioblastoma C6 cells. PTX-Tf-NPs were successfully endocytosed by $\mathrm{C} 6$ cells, and were localized in lysosomes. These data indicated that Tf-NPs can facilitate the entrance of PTX into C6 cells via transferrin-mediated endocytosis and as a result, improve the efficacy of PTX. Therefore, the present study laid the foundation for subsequent investigation of Tf-NPs as a potential nanocarrier in animal models of glioblastoma.

\section{Acknowledgements}

Not applicable.

\section{Funding}

The present study was funded by the National Natural Science Foundation of China (grant no. 81472850), Strategic Priority Research Program of the Chinese Academy of Sciences (grant no. XDA09030301), Heilong Jiang Natural Science Foundation (grant no. H2015077), Graduate Science \& Technology Research Project of Jiamusi University (grant no. Sjz2012-16) and Graduate Science \& Technology Innovation Project of Jiamusi University (grant no. LZR2014_016).

\section{Availability of data and materials}

All data generated or analyzed during the present study are included in this published article.

\section{Authors' contributions}

YW and JL conceived the study; LW designed the experiments and wrote the manuscript; LW, CL, FQ and ML performed the experiments; $\mathrm{HX}$ and $\mathrm{NC}$ conducted statistics analysis; YW and JL reviewed the experimental data and revised the manuscript critically. YW and JL approved the final manuscript. YW and JL are accountable for all aspects of the work in this study. All authors read and approved the final version of the manuscript. LW, YW and JL confirm the authenticity of all the raw data.

\section{Ethics approval and consent to participate}

Not applicable.

\section{Patient consent for publication}

Not applicable.

\section{Competing interests}

The authors declare that they have no competing interests. 


\section{References}

1. Ostrom QT, Gittleman H, Fulop J, Liu M, Blanda R, Kromer C, Wolinsky Y, Kruchko C and Barnholtz-Sloan JS: CBTRUS Statistical Report: Primary Brain and Central Nervous System Tumors Diagnosed in the United States in 2008-2012. Neuro-oncol 17 (Suppl 4): iv1-iv62, 2015.

2. Zhang X, Zhang W, Cao WD, Cheng $G$ and Zhang YQ: Glioblastoma multiforme: Molecular characterization and current treatment strategy (Review). Exp Ther Med 3: 9-14, 2012.

3. Magrath JW and Kim Y: Salinomycin's potential to eliminate glioblastoma stem cells and treat glioblastoma multiforme (Review). Int J Oncol 51: 753-759, 2017.

4. Jovčevska I, Kočevar N and Komel R: Glioma and glioblastoma - how much do we (not) know? Mol Clin Oncol 1: 935-941, 2013

5. Louis DN, Ohgaki H, Wiestler OD, Cavenee WK, Burger PC, Jouvet A, Scheithauer BW and Kleihues P: The 2007 WHO classification of tumours of the central nervous system. Acta Neuropathol 114: 97-109, 2007.

6. Stupp R, Taillibert S, Kanner AA, Kesari S, Steinberg DM, Toms SA, Taylor LP, Lieberman F, Silvani A, Fink KL, et al: Maintenance Therapy With Tumor-Treating Fields Plus Temozolomide vs Temozolomide Alone for Glioblastoma: A Randomized Clinical Trial. JAMA 314: 2535-2543, 2015.

7. Toms SA and Tapinos N: Recent Advances in the Treatment of Gliomas - Comprehensive Brain Tumor Center. R I Med J (2013) 100: 43-46, 2017

8. Chakroun RW, Zhang $\mathrm{P}$, Lin R, Schiapparelli P, Quinones-Hinojosa A and Cui H: Nanotherapeutic systems for local treatment of brain tumors. Wiley Interdiscip Rev Nanomed Nanobiotechnol 10: e1479, 2018.

9. Bryukhovetskiy IS, DyuizenIV,Shevchenko VE, Bryukhovetskiy AS Mischenko PV, Milkina EV and Khotimchenko YS: Hematopoietic stem cells as a tool for the treatment of glioblastoma multiforme. Mol Med Rep 14: 4511-4520, 2016.

10. Bryukhovetskiy I, Bryukhovetsky A, Khotimchenko Y, Mischenko P, Tolok E and Khotimchenko R: Combination of the multipotent mesenchymal stromal cell transplantation with administration of temozolomide increases survival of rats with experimental glioblastoma. Mol Med Rep 12: 2828-2834, 2015.

11. Preusser M, de Ribaupierre S, Wöhrer A, Erridge SC, Hegi M, Weller M and Stupp R: Current concepts and management of glioblastoma. Ann Neurol 70: 9-21, 2011.

12. Bryukhovetskiy I, Ponomarenko A, Lyakhova I, Zaitsev S, Zayats Y,Korneyko M,Eliseikina M, Mischenko P, Shevchenko V, Shanker Sharma $\mathrm{H}$, et al: Personalized regulation of glioblastoma cancer stem cells based on biomedical technologies: From theory to experiment (Review). Int J Mol Med 42: 691-702, 2018.

13. Hundsberger T, Reardon DA and Wen PY: Angiogenesis inhibitors in tackling recurrent glioblastoma. Expert Rev Anticancer Ther 17: 507-515, 2017.

14. Kim SS, Harford JB, Pirollo KF and Chang EH: Effective treatment of glioblastoma requires crossing the blood-brain barrier and targeting tumors including cancer stem cells: The promise of nanomedicine. Biochem Biophys Res Commun 468: 485-489, 2015.

15. Karim R, Palazzo C, Evrard B and Piel G: Nanocarriers for the treatment of glioblastoma multiforme: Current state-of-the-art. J Controll Release 227: 23-37, 2016.

16. Glaser T, Han I, Wu L and Zeng X: Targeted Nanotechnology in Glioblastoma Multiforme. Front Pharmacol 8: 166, 2017.

17. Fang K, Liu P, Dong S, Guo Y, Cui X, Zhu X, Li X, Jiang L, Liu T and $\mathrm{Wu} \mathrm{Y}$ : Magnetofection based on superparamagnetic iron oxide nanoparticle-mediated low lncRNA HOTAIR expression decreases the proliferation and invasion of glioma stem cells. Int J Oncol 49: 509-518, 2016.

18. Lee JK, Nam DH and Lee J: Repurposing antipsychotics as glioblastoma therapeutics: Potentials and challenges. Oncol Lett 11: 1281-1286, 2016.

19. Tivnan A, Zakaria Z, O'Leary C, Kögel D, Pokorny JL, Sarkaria JN and Prehn JH: Inhibition of multidrug resistance protein 1 (MRP1) improves chemotherapy drug response in primary and recurrent glioblastoma multiforme. Front Neurosci 9: 218, 2015

20. Zhao C, Liu X, Liu J, Yang Z, Rong X, Li M, Liang X and Wu Y: Transferrin conjugated poly ( $\gamma$-glutamic acid-maleimide-coL-lactide)-1,2-dipalmitoylsn-glycero-3-phosphoethanolamine copolymer nanoparticles for targeting drug delivery. Colloids Surf B Biointerfaces 123: 787-796, 2014.
21. Pierzyńska-Mach A, Janowski PA and Dobrucki JW: Evaluation of acridine orange, LysoTracker Red, and quinacrine as fluorescent probes for long-term tracking of acidic vesicles. Cytometry A 85: 729-737, 2014.

22. Choudhury H, Pandey M, Chin PX, Phang YL, Cheah JY, Ooi SC, Mak KK, Pichika MR, Kesharwani P, Hussain Z, et al: Transferrin receptors-targeting nanocarriers for efficient targeted delivery and transcytosis of drugs into the brain tumors: A review of recent advancements and emerging trends. Drug Deliv Transl Res 8: 1545-1563, 2018.

23. Gkouvatsos K, Papanikolaou G and Pantopoulos K: Regulation of iron transport and the role of transferrin. Biochim Biophys Acta 1820: 188-202, 2012.

24. Skjørringe T, Burkhart A, Johnsen KB and Moos T: Divalent metal transporter 1 (DMT1) in the brain: Implications for a role in iron transport at the blood-brain barrier, and neuronal and glial pathology. Front Mol Neurosci 8: 19, 2015.

25. Yan F,Wang Y,He S, Ku S, Gu W and YeL: Transferrin-conjugated, fluorescein-loaded magnetic nanoparticles for targeted delivery across the blood-brain barrier. J Mater Sci Mater Med 24: 2371-2379, 2013

26. Uchida Y, Ohtsuki S, Katsukura Y, Ikeda C, Suzuki T, Kamiie J and Terasaki T: Quantitative targeted absolute proteomics of human blood-brain barrier transporters and receptors. J Neurochem 117: 333-345, 2011.

27. Wiley DT, Webster P, Gale A and Davis ME: Transcytosis and brain uptake of transferrin-containing nanoparticles by tuning avidity to transferrin receptor. Proc Natl Acad Sci USA 110: 8662-8667, 2013

28. Clark AJ and Davis ME: Increased brain uptake of targeted nanoparticles by adding an acid-cleavable linkage between transferrin and the nanoparticle core. Proc Natl Acad Sci USA 112: 12486-12491, 2015.

29. Sonali, Singh RP, Singh N, Sharma G, Vijayakumar MR, Koch B, Singh S, Singh U, Dash D, Pandey BL, et al: Transferrin liposomes of docetaxel for brain-targeted cancer applications: Formulation and brain theranostics. Drug Deliv 23: 1261-1271, 2016.

30. Recht L, Torres CO, Smith TW, Raso V and Griffin TW: Transferrin receptor in normal and neoplastic brain tissue: Implications for brain-tumor immunotherapy. J Neurosurg 72: 941-945, 1990.

31. Voth B, Nagasawa DT, Pelargos PE, Chung LK, Ung N, Gopen Q, Tenn S, Kamei DT and Yang I: Transferrin receptors and glioblastoma multiforme: Current findings and potential for treatment. J Clin Neurosci 22: 1071-1076, 2015.

32. Abe T, Hasegawa S, Taniguchi K, Yokomizo A, Kuwano T, Ono M, Mori T, Hori S, Kohno K and Kuwano M: Possible involvement of multidrug-resistance-associated protein (MRP) gene expression in spontaneous drug resistance to vincristine, etoposide and adriamycin in human glioma cells. Int J Cancer 58 860-864, 1994

33. Garrido W, Muñoz M, San Martín R and Quezada C: FK506 confers chemosensitivity to anticancer drugs in glioblastoma multiforme cells by decreasing the expression of the multiple resistance-associated protein-1. Biochem Biophys Res Commun 411: 62-68, 2011.

34. Peigñan L, Garrido W, Segura R, Melo R, Rojas D, Cárcamo JG, San Martín R and Quezada C: Combined use of anticancer drugs and an inhibitor of multiple drug resistance-associated protein-1 increases sensitivity and decreases survival of glioblastoma multiforme cells in vitro. Neurochem Res 36: 1397-1406, 2011.

35. Pang Z, Gao H, Yu Y, Guo L, Chen J, Pan S, Ren J, Wen Z and Jiang $\mathrm{X}$ : Enhanced intracellular delivery and chemotherapy for glioma rats by transferrin-conjugated biodegradable polymersomes loaded with doxorubicin. Bioconjug Chem 22 1171-1180, 2011.

36. Liu G, Mao J, Jiang Z, Sun T, Hu Y, Jiang Z, Zhang C, Dong J, Huang Q and Lan Q: Transferrin-modified Doxorubicin-loaded biodegradable nanoparticles exhibit enhanced efficacy in treating brain glioma-bearing rats. Cancer Biother Radiopharm 28: 691-696, 2013.

37. Porru M, Zappavigna S, Salzano G, Luce A, Stoppacciaro A, Balestrieri ML, Artuso S, Lusa S, De Rosa G, Leonetti C, et al: Medical treatment of orthotopic glioblastoma with transferrin-conjugated nanoparticles encapsulating zoledronic acid. Oncotarget 5: 10446-10459, 2014.

38. Li Y, He H, Jia X, Lu WL, Lou J and Wei Y: A dual-targeting nanocarrier based on poly(amidoamine) dendrimers conjugated with transferrin and tamoxifen for treating brain gliomas. Biomaterials 33: 3899-3908, 2012. 
39. KhalilIR,Burns AT,RadeckaI,KowalczukM,KhalafT,Adamus G, Johnston B and Khechara MP: Bacterial-Derived Polymer Poly-y-Glutamic Acid (y-PGA)-Based Micro/Nanoparticles as a Delivery System for Antimicrobials and Other Biomedical Applications. Int J Mol Sci 18: 313, 2017.

40. Pavot V, Berthet M, Rességuier J, Legaz S, Handké N, Gilbert SC, Paul S and Verrier B: Poly(lactic acid) and poly(lactic-co-glycolic acid) particles as versatile carrier platforms for vaccine delivery. Nanomedicine (Lond) 9: 2703-2718, 2014.
41. Navarro G, Essex S, Sawant RR, Biswas S, Nagesha D, Sridhar S, de ILarduya CT and Torchilin VP: Phospholipid-modified polyethylenimine-based nanopreparations for siRNA-mediated gene silencing: Implications for transfection and the role of lipid components. Nanomedicine (Lond) 10: 411-419, 2014

42. Xiao K, Li Y, Luo J, Lee JS, Xiao W, Gonik AM, Agarwal RG and Lam KS: The effect of surface charge on in vivo biodistribution of PEG-oligocholic acid based micellar nanoparticles. Biomaterials 32: 3435-3446, 2011.

This work is licensed under a Creative Commons Attribution-NonCommercial-NoDerivatives 4.0 International (CC BY-NC-ND 4.0) License. 